Discussion: Universities should strengthen the training for undergraduates to improve their first aid skills. This is a feasible approach to promote a public level of first aid knowledge.

Prehosp Disaster Med 2019;34(Suppl. 1):s143-s144

doi:10.1017/S1049023X19003182

Issues of Which Organization is Responsible for Hospital Evacuation in Nuclear Disasters

Dr. Masaru Ogasawara ${ }^{1}$, Dr. Yuta Sato ${ }^{1}$, Dr. Katsunori Ito ${ }^{1}$, Dr. Kyoji Saito ${ }^{1}$, Dr. Katsubiro Ito ${ }^{2}$, Prof. Hiroyuki Hanada ${ }^{2}$

1. Aomori Prefectural Central Hospital, Aomori-shi, Japan

2. Hirosaki University, Hirosaki-shi, Japan

Introduction: At the time of a nuclear disaster, residents should evacuate from areas with high air dose rate. In the Great East Japan Earthquake, about $10 \%$ of patients died in a hospital evacuation in which medical teams were not involved in transportation.

Aim: To determine if hospital evacuation improved after the Fukushima nuclear accident.

Methods: This research investigates how the medical system of a nuclear disaster in Japan changed.

Results: There are 41 hospitals designated as Nuclear Emergency Core Hospitals, and they have 53 Nuclear Emergency Medical Assistance Teams (NEMAT; disaster medical dispatching team specialized in nuclear disasters consisting of medical doctors, nurses, and radiological technologists) that can support hospitals and information in the acute phase.

Discussion: At the time of a nuclear disaster, NEMAT is supposed to evacuate residents from the Urgent Protective Action Planning Zone (UPZ; within about $30 \mathrm{~km}$ radius). Tens of thousands to one million people live in this area. Hospital evacuation of more than several thousand patients is necessary. The entry of workers for transportation vehicles and lifeline restoration is limited within UPZ, so staying in a hospital is virtually impossible. There are over 2000 Disaster Medical Assistance Teams (DMAT), and many Red Cross Relief Teams; both of which are stipulated not to conduct clinical treatment in high dose areas and are not educated on nuclear disasters. Although there are Radiation Emergency Medical Assistance Teams (REMAT) consisting of doctors and technicians specializing in radiation medicine, they are few in number. They can perform dose assessment, but general medical care cannot be performed because an emergency physician is not included. Therefore, although NEMATs will conduct emergency and hospital evacuation in the affected area, the number of teams is too small to respond. The issue of which organization is responsible for massive hospital evacuation remains unsolved.

Prehosp Disaster Med 2019;34(Suppl. 1):s144

doi:10.1017/S1049023X19003194

\author{
The Knowledge, Attitudes, and Practices of Search and \\ Rescue Teams of Sri Lanka Army Regarding Search and \\ Rescue as a Response to Disasters \\ Dr. Saminda Kumara, Dr. Saveen Semage \\ Sri Lanka Army Health Services, Gampaha, Sri Lanka
}

Introduction: Sri Lanka Army is a valuable resource for the country as a capacity factor in disaster situations. Sri Lanka Army has established Search and Rescue teams (SAR teams) in all 25 districts.

Aim: To describe the knowledge, attitudes, and practices of SAR teams regarding search and rescue as a response to disasters.

Methods: A descriptive cross-sectional study was carried out from October to December 2017. Five platoons were selected randomly from high and medium risk district categories, and all five platoons were selected from the low-risk category. The total sample size was 465 . A pre-tested self-administered questionnaire was employed.

Results: The median age was 28 years. $3.2 \%$ were officers, $96.8 \%$ were soldiers, the majority $(80.4 \%)$ were educated up to G.C.E. (O/L), and $62.4 \%$ were married. $52.9 \%$ of the population had undergone SAR training during the past three years in Sri Lanka, and none had undergone training outside of the country. Overall knowledge regarding search and rescue as a response to disasters seem to be good (57.2\% received higher than a score of $75 \%$ ). $93.8 \%$ has desirable positive attitudes. $73.5 \%$ had participated in search and rescue operation as a response to disasters. Overall practices seemed to be poor, (71.3\% of the population received lower than a score of 75\%). A statistically significant association was observed with a level of education $(p=0.001)$, designation $(p=0.004)$, and knowledge on search and rescue as a response to disasters. Level of education, designation, and SAR training had no significant association with attitudes on search and rescue as a response to disasters. A statistically significant association was observed with designation $(p=0.021)$ and practices.

Discussion: Search and rescue drills should be carried out regularly. Knowledge of search and rescue as a response to disasters should be incorporated into training programs for officers and soldiers.

Prehosp Disaster Med 2019;34(Suppl. 1):s144

doi:10.1017/S1049023X19003200

Lack of Prioritization Causes Extended Time to Assessment of Severely Injured Trauma Patients in a Resource-Scarce Emergency Department

Dr. Maria Lampi ${ }^{1}$, MD John Tabu ${ }^{2}$, Dr. Johan Junker ${ }^{1}$, Professor Andreas Wladis $^{1}$

1. Centre for Teaching and Research in Disaster Medicine \& Traumatology, Linkoping, Sweden

2. Moi University College of Health and Science, Department of Disaster Risk Management, Eldoret, Kenya

Introduction: The time between injury and medical intervention is crucial in trauma care. Triage is essential to ensure prioritization and timely assessment of injured patients.

Aim: To investigate how the lack of triage system impacts timely intervention in a sub-Saharan hospital emergency department, and to investigate potential benefits of triage towards efficient management of trauma patients.

Methods: A prospective study including adult trauma patients admitted to the emergency department at Moi Teaching and Referral Hospital in Eldoret, Kenya, was conducted. Mode 
of arrival, vital parameters, time before physician's assessment, and mortality were registered. Retrospectively, Injury Severity Score (ISS) was calculated, and patients were categorized according to the Rapid Emergency Triage and Treatment System (RETTS).

Results: A total of 571 patients were analyzed, revealing a mean ISS of 12.2 (SD 7.7) and a mean length of stay of 11.6 (SD 18.3) days. 70\% of the patients arrived by taxi, private car, or police car; only $17.6 \%$ were transported by ambulance. RETTS categorization was compared with ISS using a Kruskal-Wallis test with Dunn's multiple comparisons post-test. A higher average ISS was found in the red category compared to other categories $(\mathrm{H}(\mathrm{df})=24.47(4)$, $\mathrm{p}<0.001)$. A Spearman correlation test between ISS and time to assessment revealed an $\mathrm{r}$ value of -0.041 $(\mathrm{p}=0.43)$.

Discussion: The results clearly illustrate a lack of correct prioritization of patients in relation to the need for timely assessment. Since there was no difference in time to assessment regardless of ISS, the need for a triage system is apparent. Currently, the implementation and evaluation of a validated triage tool at the emergency department are underway. Moreover, the finding that less than $18 \%$ of trauma patients are transported to the emergency department by ambulance illustrates the need to develop prehospital care systems.

Prehosp Disaster Med 2019;34(Suppl. 1):s144-s145

doi:10.1017/S1049023X19003212

Leadership and Factors Enabled the "Group Allocation" which Preserved Pre-existing Local Social Ties in Prefabricated Temporary Housing After Great East Japan Earthquake (GEJE)

Dr. Kanako Masuno ${ }^{1}$, Dr. Ken Osaka ${ }^{2}$, Dr. Jun Aida ${ }^{2}$,

$M r$. Yuichi Uchiumi ${ }^{3}, M r$. Tsuneaki Iguchi,

Dr. Yoshiyuki Hirono ${ }^{5}$

1. Showa Women's University, Sendagi, Bunkyo-ku, Japan

2. Department of International and Community Oral Health, Tohoku University Graduate School of Dentistry, Sendai, Japan

3. Department of Social Welfare, Iwanuma City (at the time of this study), Iwanuma, Japan

4. The Mayor, Iwanuma City (at the time of this study), Iwanuma, Japan

5. Department of History and Philosophy of Science, the University of Tokyo, Meguro, Japan

Introduction: Social isolation and death alone in the prefabricated temporary housing after a disaster has been a social concern. The importance of social ties among the community has been suggested and several reports pointed out the positive effect of "group allocation" which preserves pre-existing local social ties compared to the "lottery allocation".

Japan Red Cross Society recommended "group allocation" as a better option than "lottery allocation" on their guidelines. However, many municipalities carried out "lottery allocation" for temporary housing arrangement after the Great East Japan Earthquake (GEJE).

Aim: To collect the information about the accelerating factors and bottlenecks when practicing the "group allocation".
Method: In-depth interview was conducted between August and November 2013. Interviewees were the professionals of disaster management, individuals who were involved in arranging the prefabricated housing and the residents. This research was supported by the Ministry of Education, Culture, Sports, Science, and Technology in Japan.

Results: This study found the municipality which carried out "group allocation" had characteristics such as: (1.) the staff in charge of housing arrangement had the information about the positive effect of "group allocation", and (2.) pre-existing community leaders were able to gather residents' opinions, and citizens were involved in the decision making to some content.

Discussion: Although this study is based on the experience of a limited number of key persons, it would be useful to give the insight about the possible bottleneck for the practitioners who will be in charge of housing arrangement under the disaster setting in future. Also, the relevancy and evidence about "group allocation" should be carefully examined in the context of preventing social isolation as well as various long-term effects. It would be essential that the knowledge and experience will be accumulated and shared between municipalities in a usable and comparable format.

Prehosp Disaster Med 2019;34(Suppl. 1):s145

doi:10.1017/S1049023X19003224

\section{Learning Effects of Cross Road Game Using a Clicker- Nano System}

Ms. Satoko Mitani

Gifu University of Medical Sciences, Seki, Gifu, Japan

Introduction: There are some tools for teaching disaster countermeasure in Japan. Cross Road Game was developed to get a concrete image of a disaster situation and is based on interviews from Kobe city government officers in an area affected by the 1995 Great Hanshin-Awaji Earthquake. The alternative includes a lot of 'dilemmas' that sacrifice something based on whichever outcome is chosen. For example, "There are 2000 meals at the evacuation center with 3000 refugees. Do you distribute these foods or not?" This game was developed for five to seven players, however, it is not suitable for class lessons with a hundred students. Thus, we tried to employ the Clicker-Nano system for an interactive lesson.

Aim: To provide a brief introduction to this new style of teaching disaster countermeasure.

Methods: The study included involved a classroom discussion using Clickers-Nano system in addition to Cross Road Game. Results: Nursing students could learn the concrete details of disaster countermeasure in an enjoyable format. They could share thoughts and compare opinions while deciding how to resolve the dilemma at the time of disaster.

Discussion: The most important issue faced was how to develop an educational effect for nursing students. Even if five or seven students (players) could enjoy the game, it would not lead to the accumulation of unified knowledge of disaster countermeasure compared to a lecture at the university. The use of the Clickers-Nano system avoided differences in the reach of 